\title{
Geometric Linearization of Ordinary Differential Equations ${ }^{\star}$
}

\author{
Asghar QADIR \\ Centre for Advanced Mathematics and Physics, National University of Sciences and Technology, \\ Campus of College of Electrical and Mechanical Engineering, \\ Peshawar Road, Rawalpindi, Pakistan \\ E-mail: aqadirmath@yahoo.com
}

Received August 13, 2007, in final form October 19, 2007; Published online November 06, 2007

Original article is available at http://www.emis.de/journals/SIGMA/2007/103/

\begin{abstract}
The linearizability of differential equations was first considered by Lie for scalar second order semi-linear ordinary differential equations. Since then there has been considerable work done on the algebraic classification of linearizable equations and even on systems of equations. However, little has been done in the way of providing explicit criteria to determine their linearizability. Using the connection between isometries and symmetries of the system of geodesic equations criteria were established for second order quadratically and cubically semi-linear equations and for systems of equations. The connection was proved for maximally symmetric spaces and a conjecture was put forward for other cases. Here the criteria are briefly reviewed and the conjecture is proved.
\end{abstract}

Key words: differential equations; geodesics; geometry; linearizability; linearization

2000 Mathematics Subject Classification: 34A34; 34A26

\section{Introduction}

Whereas there are standard methods to solve linear ordinary differential equations (ODEs), there are hardly any for nonlinear ODEs. One could, of course, approximate the nonlinear ODE by a linear one (as in perturbation methods), but the key features of the nonlinear equation that may be vital for the phenomenon being modeled could easily be lost. As such, one needs methods to obtain exact solutions of the equation. Further, if one tries to improve the approximation by an iterative series the convergence of the series would need to be proved. The same problem is faced by numerical schemes for solution. Proof of existence and convergence is provided by functional analytic methods when they can be applied. On the other hand, some methods were developed for specific classes of first order ODEs and some second order ODEs were converted to linear form by transformation of variables. Using symmetry considerations, Lie had found that all first order ODEs could, in principle, be converted to linear form by some appropriate change of independent and dependent variables. (The infinitesimal generators of symmetry form a Lie algebra [3].) He obtained criteria for a second order semi-linear ODE to be such that it could be converted to linear form by point transformations [1, 2]. He did not go further to deal with systems of ODEs or third order ODEs. His procedure uses transformations of the dependent and independent variables of the ODE (or system of ODEs) There has been work done on obtaining the symmetry classes of systems of second order ODEs [4] and for the third order ODE [5]. Some explicit criteria for the linearizability of single third order ODEs have also been provided in the literature $[6,7,8,9,11,12]$.

${ }^{\star}$ This paper is a contribution to the Proceedings of the Seventh International Conference "Symmetry in Nonlinear Mathematical Physics" (June 24-30, 2007, Kyiv, Ukraine). The full collection is available at http://www.emis.de/journals/SIGMA/symmetry2007.html 
It is natural to ask what the connection is between symmetries in geometry and for differential equations. After all, differential equations "live" on manifolds. More precisely, we would like to relate the symmetry algebra of the ODEs to that of the manifold. There had been some suggestions but there does not appear to have been any attempt to formulate the connection precisely till the turn of the century. The first such attempt looked for the connection through the system of geodesic equations[13] $]^{1}$. It was used for the projective geometry of geodesics. (A recent paper [14], and references therein, detail relations between projective algebra (point symmetries of a particular class of second order ODEs) and algebra of isometries, providing a more complete background of geometry for the purpose.) Independently, the same idea was developed and carried further to construct the Lie algebra of the system of geodesic equations on maximally symmetric spaces [15]. There a conjecture was stated for all spaces. In this paper that conjecture will be proved.

The plan of the paper is as follows. A brief review of the geometrical notation used will be presented in the next section. The application of the geometric method for linearization of second order ODEs will be presented in the subsequent section and its extension to higher orders in Section 4. The theorem will be proved in the subsequent section and a brief summary and discussion presented in Section 6 .

\section{Geometric notation used}

To explain the linearization procedure it is necessary to introduce the geometric notation used. This will be presented first.

The following are well-known and can be found in text books. We use the Einstein summation convention that repeated indices are summed over the entire range of the index. Thus, $A^{a} B_{a}$ stands for $\sum_{a=1}^{n} A^{a} B_{a}$. The metric tensor will be represented by the symmetric (nonsingular) matrix $g_{i j}$ and its inverse by $g^{i j}$. The Christoffel symbols are given by

$$
\Gamma_{j k}^{i}=\frac{1}{2} g^{i m}\left(g_{j m, k}+g_{k m, j}-g_{j k, m}\right),
$$

where, $k$ stands for partial derivative relative to $x^{k}$, etc. The Christoffel symbols are symmetric in the lower pair of indices, $\Gamma_{j k}^{i}=\Gamma_{k j}^{i}$.

In this notation, the system of $n$ geodesic equations is

$$
\ddot{x}^{i}+\Gamma_{j k}^{i} \dot{x}^{j} \dot{x}^{k}=0, \quad i, j, k=1, \ldots, n,
$$

where $\dot{x}^{i}$ is the derivative relative to the arc length parameter $s$ defined by $d s^{2}=g_{i j} d x^{i} d x^{j}$.

The Riemann tensor is defined by

$$
R_{j k l}^{i}=\Gamma_{j l, k}^{i}-\Gamma_{j k, l}^{i}+\Gamma_{m k}^{i} \Gamma_{j l}^{m}-\Gamma_{m l}^{i} \Gamma_{j k}^{m},
$$

and has the properties $R_{j k l}^{i}=-R_{j l k}^{i}, R_{j k l}^{i}+R_{k l j}^{i}+R_{l j k}^{i}=0$ and $R_{j k l ; m}^{i}+R_{j l m ; k}^{i}+R_{j m k ; l}^{i}=0$. The Riemann tensor in fully covariant form $R_{i j k l}=g_{i m} R_{j k l}^{m}$ satisfies $R_{i j k l}=-R_{j i k l}$.

\section{$3 \quad$ Linearizability of second order ODEs}

A general quadratically semi-linear system of ODEs is of the form

$$
x^{a \prime \prime}+\alpha^{a}+\beta_{b}^{a} x^{b^{\prime}}+\gamma_{b c}^{a} x^{b^{\prime}} x^{c \prime}=0 .
$$

\footnotetext{
${ }^{1}$ There appears to be a Russian preprint of 1991, but that is even less accessible than this paper.
} 
The system of ODEs will be said to be of geodesic form if $\alpha^{a}=\beta_{b}^{a}=0$. It can be regarded as a system of geodesic equations if there exists some metric tensor for which the Christoffel symbols satisfy $\Gamma_{b c}^{a}=\gamma_{b c}^{a}$. In this case we can construct the curvature tensor corresponding to the given coefficients. It is easier to see the procedure adopted for the case of two dependent variables. Call the variables $x, y$. Then we have 6 arbitrary functions of these variables appearing as coefficients in the equations

$$
\begin{aligned}
& x^{\prime \prime}=a(x, y) x^{\prime 2}+2 b(x, y) x^{\prime} y^{\prime}+c(x, y) y^{\prime 2}, \\
& y^{\prime \prime}=d(x, y) x^{2}+2 e(x, y) x^{\prime} y^{\prime}+f(x, y) y^{\prime 2},
\end{aligned}
$$

the Christoffel symbols in terms of these coefficients are

$$
\Gamma_{11}^{1}=-a, \quad \Gamma_{12}^{1}=-b, \quad \Gamma_{22}^{1}=-c, \quad \Gamma_{11}^{2}=-d, \quad \Gamma_{12}^{2}=-e, \quad \Gamma_{22}^{2}=-f .
$$

The linearizability criteria are [16]

$$
\begin{aligned}
& a_{y}-b_{x}+b e-c d=0, \quad b_{y}-c_{x}+\left(a c-b^{2}\right)+(b f-c e)=0, \\
& d_{y}-e_{x}-(a e-b d)-\left(d f-e^{2}\right)=0, \quad(b+f)_{x}=(a+e)_{y},
\end{aligned}
$$

with constraints on the metric coefficients: $g_{11}=p, g_{12}=g_{21}=q, g_{22}=r$,

$$
\begin{array}{llrl}
p_{x} & =-2(a p+d q), & q_{x}=-b p-(a+e) q-d r, & r_{x}=-2(b q+e r), \\
p_{y}=-2(b p+e q), & q_{y}=-c p-(b+f) q-e r, & r_{y}=-2(c q+f r) .
\end{array}
$$

The compatibility of this set of six equations gives the above four linearization conditions (2). The remarkable fact is that the linearization criteria are simply $R_{j k l}^{i}=0$ ! For a system of three equations we get eighteen such equations. In general there are $n^{2}(n+1) / 2, n \geq 2$. One now obtains the required linearizing transformation by regarding the variables in which the equation is linearized as Cartesian, thus having $g_{11}=g_{22}=1$ and $g_{12}=g_{21}=0$, and looking for the coordinate transformation yielding the original metric coefficients [16]. This can be done by using complex variables. The details can be found in the reference, where examples are also given.

Following Aminova and Aminov [13] and projecting the system $n$-dimensional system down to a system of $(n-1)$ dimensions, the geodesic equations become [17]

$$
x^{a \prime \prime}+A_{b c} x^{a \prime} x^{b^{\prime}} x^{c^{\prime}}+B_{b c}^{a} x^{b^{\prime}} x^{c^{\prime}}+C_{b}^{a} x^{b^{\prime}}+D^{a}=0, \quad a=2, \ldots, n,
$$

where the prime now denotes differentiation with respect to the parameter $x^{1}$ and the coefficients in terms of the $\Gamma_{b c}^{a}$ s are

$$
\begin{aligned}
& A_{b c}=-\Gamma_{b c}^{1}, \quad B_{b c}^{a}=\Gamma_{b c}^{a}-2 \delta_{(c}^{a} \Gamma_{b) 1}^{1}, \\
& C_{b}^{a}=2 \Gamma_{1 b}^{a}-\delta_{b}^{a} \Gamma_{11}^{1}, \quad D^{a}=\Gamma_{11}^{a}, \quad a, b, c=2, \ldots, n .
\end{aligned}
$$

System (3) is cubically semi-linear. When $n=2$, writing $a-2 e=h, f-2 b=g$, we get

$$
y^{\prime \prime}+c(x, y) y^{\prime 3}-g(x, y) y^{\prime 2}+h(x, y) y^{\prime}-d(x, y)=0 .
$$

This is the general form of linearizable second order semi-linear ODE found by Lie, with the coefficients re-named. To be linearizable it must satisfy the linearizability conditions

$$
\begin{aligned}
& h_{y}+2 e_{y}-b_{x}+b e-c d=0, \quad b_{y}-c_{x}+h c+e c+b^{2}-b g=0, \\
& -d_{y}+e_{x}+e h+e^{2}+b d-d g=0, \quad 3 b_{x}-3 e_{y}-g_{x}-h_{y}=0,
\end{aligned}
$$

which are simply the Lie criteria for a scalar ODE! 
One can use the same procedure to go on to systems of two (or more) cubically semi-linear ODEs by taking $n \geq 3$ in (1). This has been done [17] and examples of the two dimensional case are also given there.

It is known that there is a unique class of linearizable scalar second order semi-linear ODEs, namely that which satisfies the Lie criteria. One may wonder if the extension provided for systems will give a unique class as well. The answer is "No", as it is known that for 2-dimensional systems there are five classes [4] that are linearizable and only one has been provided by the above procedure. How is it that the case of the single equation is fully covered by the above procedure but the systems are not fully covered?

Note that the projection procedure applied to an arbitrary system of $n$ quadratically semilinear second order ODEs reduces it to $n-1$ cubically semi-linear second order ODEs. (Of course there is an enormous computational complication arising. To construct the metric tensor from the Christoffel coefficients an algebraic computational code has been written [18].) Now observe that in projecting down from the system of $n$ variables to $n-1$ variables, the Christoffel symbols are reduced from $n^{2}(n+1) / 2$ by $n$, to give $(n-1) n(n+2) / 2$ independent coefficients. Since we now have $n-1$ equations, each with its own cubic function, there are $4(n-1)$ coefficients for the reduced system. If the number of coefficients left after losing $n$ equals the number of coefficients of the reduced system, we can determine one set of coefficients in terms of the other. The two expressions are obviously equal for $n=2$ and the former is greater than the latter for $n>2$. As such, the coefficients of the cubic system can be determined uniquely in terms of the quadratic system for $n=2$, i.e. for a scalar cubically semi-linear system. For larger systems there will be infinitely many ways to write the former in terms of the latter. This is why there is a unique solution to the linearizability problem for the scalar equation and many solutions for the systems! It can be seen that (3) is not the most general form for the system as one should have $A_{b c d}^{a} x^{b^{\prime}} x^{c \prime} x^{d^{\prime}}$ in place of the second term for full generality. For $n=2$ there can be no new term coming from this more general coefficient, but for $n \geq 3$ new terms will come in. This is where the extra classes that are missed for $n \geq 3$ will come from.

\section{Higher order systems}

A procedure to extend the scalar second order case to the third order is to differentiate the general second order ODE (4). One can now replace the second order derivative term in the equation by using the original second order ODE. Thus [19] one either gets a third order ODE which is quintic in the first derivative

$$
y^{\prime \prime \prime}-\alpha y^{\prime 5}+\beta y^{\prime 4}-\gamma y^{\prime 3}+\delta y^{\prime 2}-\epsilon y^{\prime}+\phi=0,
$$

or a third order ODE with a term in the second order that has a coefficient quadratic in the first derivative

$$
\begin{aligned}
y^{\prime \prime \prime}- & \left(A(x, y) y^{\prime 2}-B(x, y) y^{\prime}+C(x, y)\right) y^{\prime \prime}+D(x, y) y^{\prime 4}+E(x, y) y^{\prime 3} \\
& -F(x, y) y^{\prime 2}+G(x, y) y^{\prime}-H(x, y)=0 .
\end{aligned}
$$

It turns out that the same result is obtained in either case. These equations are not in the classes considered in the literature earlier. It must be added that the class of equations obtained by this procedure also does not contain the classes earlier discussed in the literature. Instead of following this procedure, one could have gone in the reverse direction. First differentiate the system and then project it. Surprisingly, it turns out that the result is again the same in either case. Some examples of linearizable third order equations are given in [19], one of which overlaps with the class of Meleshko [12], but none of which overlaps with that of Ibragimov and Meleshko [11]. 
It should be noted that if one tries to reduce from the quintic, it is not possible to reduce to the quartic. Further, the cubic cannot be resolved directly as there are problems of nonuniqueness of the solutions of the equations for the coefficients required to linearize the third order cubic ODE. It would be useful to find a canonical way to solve the equations, or to prove that all solutions are equivalent. One needs to understand how a complete classification of the various classes can be obtained and to provide explicit criteria and constructive procedures for the solutions. In particular, there are only three classes possible for the third order ODEs linearizable by point transformations [5], $y^{\prime \prime \prime}(x)=0, y^{\prime \prime \prime}(x)=y(x)$ and $y^{\prime \prime \prime}(x)=\alpha(x) y(x)$. Two were provided by Chern [6, 7] and the third by Neut and Petitot [10], and later by Ibragimov and Meleshko [11]. How can there then be another class? The point is that the method just outlined uses a projection and differentiation apart from the point transformations. It guarantees only two arbitrary constants and not three while the other procedure guarantees three.

Can one go further? On differentiating the third order ODE (in the quintically semi-linear form) and using the second order ODE, just as in the case of the third order cubically semi-linear ODE, there is no guarantee that the coefficients of the fourth order ODE would be resolvable in terms of the coefficients of the original second order ODE. It turns out that it is [20] resolvable. However, as yet there is no proof that the other forms of the equation, involving the third and lower orders or the second and first orders, would yield the same result. It certainly is not clear that the projection and differentiation procedures would commute. It is not even clear how that would be checked. One would need a system of third order equations to be able to check this result. Though the system of third order equations that are cubically semi-linear in the the first derivative has been computed [21], it is highly complicated and one cannot yet make any statement about the commutation of the different procedures.

\section{Proof of the Feroze-Mahomed-Qadir conjecture}

In [15] maximal curvature spaces were considered and it was found that for flat spaces of dimension $n$ the symmetry algebra is $s l(n+2, \mathbb{R})$, for positive curvature $s o(n+1)$, or for negative curvature a corresponding non-compact version. There was no result for spaces that are not maximally symmetric. However, one can imagine some invariant procedure to split the space into flat, positive or negative curvature spaces, or those with no symmetry. Considering each of these subspaces it seemed clear that the corresponding direct sums should provide the full symmetry algebra. In case there is no flat subspace, there will still remain the geodesic re-parametrization by translation and scaling. This is what gives the extra 2 in $\operatorname{sl}(n+2, \mathbb{R})$. Where there is no part of the special linear algebra the dilation algebra, $d_{2}$, will replace it. On this basis a more general conjecture was stated. That conjecture is now proved as a theorem.

Theorem. For a space of non-zero curvature with isometry algebra $h$ the symmetry algebra of the geodesic equations is $h \oplus d_{2}$ provided that there is no section of zero curvature. If there is an m-dimensional maximal cross-section of zero curvature, $M$, and the symmetry algebra of the orthogonal subspace, $M^{\perp}$ is $h_{1}$, the symmetry algebra of the geodesic equations will be $h_{1} \oplus \operatorname{sl}(m+2, \mathbb{R})$.

Proof. First consider a base manifold $N$, which has a maximal flat section $M$ of dimension $m \neq 0$ such that $N=M \times M^{\perp}$, where the orthogonal subspace, $M^{\perp}$, has no flat section. Consider a metric tensor $\mathrm{g}$ on $N$ and let the isometry algebra on $M^{\perp}$ be $h$. Thus the isometry algebra of $N$ will be $s o(m) \oplus_{s} \oplus h$, where $\oplus_{s}$ denotes the semi-direct sum, i.e. a "non-commutative direct sum".

Construct a fibre bundle on $N$ with the fibre given by $\mathbb{R}$ and take the position vector $\mathbf{x}$ to depend on the arc length, or geodetic, parameter along the fibre $s$, i.e. such that $\mathbf{g} . \dot{\mathbf{x}} \dot{\mathbf{x}}$ is unity. If one defines the Lagrangian, as a function of $\mathbf{x}$ and $\dot{\mathbf{x}}$ to be this quantity, it will possess at least 
one additional symmetry generator, $\partial / \partial s$, apart from the isometry algebra. The Lagrangian is not, in general, invariant under the generator $s \partial / \partial s$ (unless it has the value zero). However, the Euler-Lagrange equations for this Lagrangian will be invariant under this additional generator.

Consider the geodesic equations for $M$ in the fibre bundle over it. They will be the vector freeparticle equations and hence will have the symmetry algebra $s l(m+2, \mathbb{R})$. The remaining part will retain its isometry algebra. Thus, in this case, the full symmetry algebra is $h_{1} \oplus s l(m+2, \mathbb{R})$.

Now consider the case that there is no flat section of the manifold, $M=\varnothing$ so $m=0$. In this case the isometry algebra will simply be $h$. There will remain the two symmetry generators for re-parametrization of the geodetic parameter by translation and re-scaling, $\partial / \partial s$ and $s \partial / \partial s$. They form a two-dimensional dilation algebra, $d_{2}$.

This theorem provides the basis for using geometric procedures to obtain linearizable forms of (systems of) ODEs, through the link between the symmetries of the manifold and the system of geodesic equations on it. In particular, one requires a flat space for the system of geodesics to be linearizable. The geometry of the projective space need not be flat but it must be imbeddable in a flat space. While it was used as a conjecture, one really needed a rigorous proof of the result.

\section{Conclusion}

In this paper a review has been provided of the geometric approach to symmetry analysis using the connection between symmetries of geometry and of differential equations provided by the system of geodesic equations, noted by Aminova and Aminov [13] and separately by Feroze et al. [15]. This leads to a new approach to investigate the linearizability of ODEs. A bonus of this approach is that the solution of the linearized equations is obtainable by constructing the transformation of coordinates from Cartesian to the metric tensor given by the geodesic equations [16]. Using this approach and the projection procedure of Aminova and Aminov [13] one can re-derive the Lie criteria for a scalar equation and extend them to a system of equations [17]. One can also extend to the third and higher order scalar equations [19] and to the third order system of equations [21]. There is much work that needs to be done in this direction. For one thing, the systems have many classes of which one segment has been dealt with so far. A complete analysis and a method to find solutions of the remaining classes would be very important. Again, the higher order equations can be linearized by using the Lie procedure as done by Ibragimov and Meleshko [11] or by the procedure of Neut and Petitot [10]. To what extent is there an overlap between the two procedures? For that matter, does this work subsume the work of Grebot $[8,9]$ or not? The relation between the various methods and approaches being used is needed for a proper understanding and classification of the procedures.

A conjecture that was at the base of the connection between geometry and symmetry analysis found by Feroze et al [15] was stated and proved as a theorem. The proof given demonstrates that there is at least one extra symmetry generator of the Lagrangian apart from the isometry algebra, and that the symmetry algebra of the geodesic equations contains the symmetry algebra of the Lagrangian. The Lagrangian can have additional symmetries beyond the one extra symmetry generator of geodetic re-parametrization by translation due to "mixing" between the additional dimension of the fibre bundle and the other flat dimensions. It would be of interest to use the above approach in proving the conjecture to determine the symmetry algebra of the Lagrangian. The problem is that whereas for the Euler-Lagrange equations the "mixing of the two symmetries of the fibre with the flat base space" is apparent and is maximal, that is not true for the Lagrangian. For one thing, there is generally only one symmetry for the fibre and the mixing along a section of the fibre is not maximal and is not apparent. How, and to what extent, the extra symmetries from the fibre come into the Lagrangian needs to be determined rigorously. 


\section{Acknowledgements}

I am grateful to NUST for travel support and to the organizers of the Symmetry-2007 and the International Mathematical Union for local support and hospitality at the conference where this paper was presented. I am also grateful for useful comments to Professors Leach, Mahomed, Meleshko and Popovych. Thanks also to DECMA and CAM of the of Wits University, Johannesburg, South Africa for support at the University where the paper was completed.

\section{References}

[1] Lie S., Theorie der transformationsgruppen, Math. Ann. 16 (1880), 441-528.

[2] Lie S., Klassification und Integration von gewöhnlichen Differentialgleichungen zwischen $x$, $y$, die eine Gruppe von Transformationen gestatten, Arch. Math. Naturv. 9 (1883), 371-393.

[3] Olver P.J., Applications of Lie groups to differential equations, Springer, New York, 1986.

[4] Wafo Soh C., Mahomed F.M., Symmetry breaking for a system of two linear second-order ordinary differential equations, Nonlinear Dynamics 22 (2000), 121-133.

[5] Mahomed F.M., Leach P.G.L., Symmetry Lie algebras of $n$th order ordinary differential equations, J. Math. Anal. Appl. 151 (1990), 80-107.

[6] Chern S.S., Sur la geometrie d'une equation differentielle du troiseme orde, C.R. Acad. Sci. Paris 204 (1937), 1227-1229.

[7] Chern S.S., The geometry of the differential equation $y^{\prime \prime \prime}=F\left(x, y, y^{\prime}, y^{\prime \prime}\right)$, Sci. Rep. Nat. Tsing Hua Univ. 4 (1940), 97-111.

[8] Grebot G., The linearization of third order ODEs, Preprint, 1996.

[9] Grebot G., The characterization of third order ordinary differential equations admitting a transitive fibrepreserving point symmetry group, J. Math. Anal. Appl. 206 (1997), 364-388.

[10] Neut S., Petitot M., La géométrie de l'équation $y^{\prime \prime \prime}=f\left(x, y, y^{\prime}, y^{\prime \prime}\right)$, C.R. Acad. Sci. Paris Sér I 335 (2002), $515-518$.

[11] Ibragimov N.H., Meleshko S.V., Linearization of third-order ordinary differential equations by point and contact transformations, J. Math. Anal. Appl. 308 (2005), 266-289.

[12] Meleshko S.V., On linearization of third-order ordinary differential equations, J. Phys. A: Math. Gen. Math. 39 (2006), 15135-15145.

[13] Aminova A.V., Aminov N.A.-M., Projective geometry of systems of differential equations: general conceptions, Tensor NS 62 (2000), 65-85.

[14] Bryant R.L., Manno G., Matveev V.S., A solution of a problem of Sophus Lie: normal forms of 2-dim metrics admitting two projective vector fields, arXiv:0705.3592.

[15] Feroze T., Mahomed F.M., Qadir A., The connection between isometries and symmetries of geodesic equations of the underlying spaces, Nonlinear Dynamics 45 (2006), 65-74.

[16] Mahomed F.M., Qadir A., Linearization criteria for a system of second-order quadratically semi-linear ordinary differential equations, Nonlinear Dynamics 48 (2007), 417-422.

[17] Mahomed F.M., Qadir A., Invariant linearization criteria for systems of cubically semi-linear second-order ordinary differential equations, arXiv:0711.1213.

[18] Fredericks E., Mahomed F.M., Momoniat E., Qadir A., Constructing a space from the system of geodesic equations, arXiv:0711.1217.

[19] Mahomed F.M., Qadir A., Linearizability criteria for a class of third order semi-linear ODEs, arXiv:0711.1214.

[20] Mahomed F.M., Qadir A., Conditional linearizability criteria for scalar fourth order semi-linear ordinary differential equations, arXiv:0711.1222.

[21] Mahomed F.M., Naeem I., Qadir A., Conditional linearizability criteria for a system of third-order ordinary differential equations, arXiv:0711.1215. 